\title{
Patients with Essential Thrombocythaemia have an Increased Prevalence of Antiphospholipid Antibodies which may be associated with Thrombosis
}

\author{
C. N. Harrison, S. Donohoe, P. Carr, M. Dave, I. Mackie, S. J. Machin \\ Department of Haematology, University College London Hospital, UK
}

\section{Keywords}

ET, antiphospholipid antibodies, thrombosis

\section{Summary}

A significant proportion of patients with Essential Thrombocythaemia (ET) have thrombotic complications which have an important impact upon the quality, and duration of their life. We performed a retrospective cross sectional study of the prevalence of antiphospholipid antibodies (APA) in 68 ET patients. Compared to 200 "elderly" controls (> 50 years) there was a significant increase in anticardiolipin $\operatorname{IgM}$ $(\mathrm{p}<0.0001)$ and anti $\beta_{2}$ glycoprotein I (anti- $\left.\beta_{2} \mathrm{GPI}\right) \operatorname{IgM}(\mathrm{p}<0.0001)$ antibodies in ET. Thrombosis occurred in 10/20 with APA and 12/48 without, $\mathrm{p}=0.04$, relative risk 2.0 (95\% confidence intervals 1.03-3.86); these patients did not differ in terms of other clinical features. The prevalence of thrombosis in patients with dual APA (6/7) was significant when compared to those with single APA $(p=0.02)$ and the remaining patients $(\mathrm{p}<0.0002)$. Also anti- $\beta_{2} \mathrm{GP1}$ IgM antibodies either alone, or in combination with another APA, were associated with thrombosis $(p=0.02)$. These results suggest that the prevalence of APA in ET and their influence upon thrombotic risk merit investigation in a larger study.

\section{Introduction}

Essential thrombocythaemia (ET) is a chronic myeloproliferative disorder (MPD) characterised by a sustained thrombocytosis, bone marrow megakaryocyte hyperplasia and in some patients thrombotic and/or haemorrhagic complications. A number of studies of patients with ET have demonstrated that it is the occurrence of disease related complications, in particular thrombotic episodes, which have significant impact upon overall survival $(1,2)$. For example Lengfelder (2) performed a retrospective analysis of 143 patients with ET, and reported that the main factor influencing survival after multivariate analysis was the number of complications occurring during follow up $(\mathrm{p}=0.002)$.

Cytoreductive treatment with drugs such as hydroxyurea has been demonstrated to reduce the incidence of thrombotic episodes especially in high risk patients (1). Unfortunately it has recently been suggested that the use of hydroxyurea might be associated with the development

Correspondence to: Dr. Claire N. Harrison, Department of Haematology, St. Thomas' Hospital, Lambeth Palace Road, London, SE1 7EH, UK Tel.: 4479289292 ext. 3504; Fax: 447928 7226; E-mail: claire.harrison@ gstt.sthames.nhs.uk of secondary myeloid malignancy and a specific chromosomal abnormality (3). This finding increases the need for risk stratification in the management of patients with ET, such that the patients at highest risk of thrombotic complications receive cytoreductive therapy and those at lowest risk do not. The appropriate risk factors for thrombotic complications in ET therefore need to be carefully defined. Previously both age exceeding 60 years and prior thrombosis $(\mathrm{p}=<0.001$ and $p<0.0005$ respectively) have been demonstrated to be associated with increased thrombotic risk; whilst smoking, hypertension and age 40-60 years were not significant risk factors in this analysis (4). We and others have previously reported that ET is a heterogeneous disorder in terms of clonality assessed by X-chromosome inactivation patterns and that those patients with monoclonal haemopoiesis appear to be at increased risk of thrombosis $(5,6)$. Other factors reported to be associated with an increased risk of thrombosis in ET include a platelet count of more than $600 \times 10^{9} / \mathrm{L}$ (4), and spontaneous megakaryocyte colonies (7).

We previously reported an apparent association in two patients with ET and anticardiolipin antibodies following an audit of patients with ET (8). This association may aid the prediction of patients at risk of thrombosis and might influence management. In the current study we performed a single point cross-sectional retrospective survey of the prevalence of immunologically detectable antiphospholipid antibodies (APA) (anticardiolipin, anti- $\beta_{2}$ glycoprotein I and anti-prothrombin) in 68 consecutive patients with ET. The prevalence of APA in ET was compared to that in 200 normal controls; clinical features, including clonality, were compared and the contribution of APA to the risk of thrombotic complications was evaluated.

\section{Patients and Methods}

Patients

Sixty eight patients with a diagnosis of ET made according to the Polycythaemia Vera Study Group criteria (9) were included in this study. Forty had been included in a previously reported study of clonal analysis (5).

Patients with ET attended a specialist clinic based within University College London Hospitals as part of their on-going care, or, in the case of fifteen of the patients when referred for a single consultation. Regular follow up is documented on all of these patients on an annual basis. Overall 21/68 patients were male, median age was 56 years (range 11-89), and platelet count at test $495 \times$ $10^{9} / \mathrm{L}$ (231-1462). Follow up to the time of analysis ranged from 1 to 202 months (median 46), 12 patients had hepato or splenomegaly, 22 thrombotic events, and 6 haemorrhagic episodes. At the time of testing for APA one patient had received no treatment due to aspirin intolerance, 22 patients aspirin alone, and 45 cytoreductive therapy with aspirin. Cytoreductive therapy included in 35 cases hydroxyurea, in 3 anagrelide, 7 interferon, 4 busulphan, 3 radioactive phosphorous, 1 chlorambucil and in 8 cases multiple agents. No patient was tested for APA within 3 months of a thrombotic event. 


\section{Normal Controls}

Normal ranges for APA in our laboratory have previously been derived from healthy young adults. In this study normal ranges were determined from samples obtained from healthy older (more than 50 years) individuals. This group were specifically selected because patients with ET are older and there is a suggestion that APA are present at a higher frequency in the elderly than young (10). The healthy older adults had participated in a long-haul air travel study in 1999-2000 (11). Passengers were excluded from this study if they had previous substantiated episodes of venous or arterial thrombosis; were taking anticoagulants (warfarin or heparin); regularly wore elasticated compression stockings; suffered from severe cardiorespiratory disease or any other serious illness including malignant diseases (11). This control population included 76 males and 124 females; median age was 62 years (range 50-86).

\section{Antiphospholipid Antibody Assays}

Anticardiolipin Antibodies ( $a C L)$ - IgG and IgM antibodies to cardiolipin were measured in serum samples by an ELISA technique based on the method of Loizou (12), using microtitre plates (Polysorp, Nunc, Life Technologies, Paisley, UK) coated with cardiolipin (bovine heart, Sigma-Aldrich Ltd. Poole, UK), and blank well subtraction. Assays were standardised using standard sera calibrated against the appropriate International Reference Material (13).

Anti- $\beta_{2} G P I$ Antibodies (Anti- $\beta_{2} G P I$ ) - Anti- $\beta_{2}$ GPI IgG was measured by ELISA, using a commercial kit (Diastat anti- $\beta_{2}$ GPI, Axis-Shield Diagnostics, Dundee, UK) based on a method previously developed in our laboratory (14). Anti- $\beta_{2}$ GPI IgM was assayed by an adaptation of the $\operatorname{IgG}$ kit components to accommodate the detection of IgM antibodies by using a $\mu$-specific conjugated secondary antibody. Assays were standardised using serum with known high concentration of antibody.

Anti-Prothrombin Antibodies ( $\alpha$-Pro) - Anti-prothrombin $\operatorname{IgG}$ and $\operatorname{IgM}$ were analysed by ELISA (15), using gamma irradiated microtitre plates (Maxisorp, Nunc, Life Technologies, Paisley, UK) coated with $10 \mu \mathrm{g} / \mathrm{ml}$ human prothrombin (Enzyme Research Laboratories, Swansea, UK) in PBS
(0.01 M phosphate, $0.145 \mathrm{M} \mathrm{NaCl}, \mathrm{pH}$ 7.2). Blocking was performed using PBS containing $0.1 \%$ Tween 20 and $1 \%$ bovine serum albumin (BSA, A-7030, Sigma-Aldrich, Poole, UK). Test samples were diluted 1:50 in PBS/Tween/ BSA and bound antibody was detected using alkaline phosphatase conjugated anti-human $\operatorname{IgG}$ or $\operatorname{IgM}$. The assay was standardised using a reference plasma with a known high anti-prothrombin activity. All assays were validated by the inclusion of internal quality control samples of known activity.

Reference ranges for all APA were established from the normal control population using a cut off of the geometrical mean plus 4 standard deviations.

\section{Statistical Analysis}

Chi squared or Mann-U-Whitney tests were performed using Astute add-in for Microsoft ${ }^{\circledR}$, Excel (DDU Software, Leeds, UK), level of significance was taken as $\mathrm{p}<0.05$.

\section{Results}

\section{Normal Controls}

Overall there were $12(6 \%)$ APA detected in the "healthy elderly" control population; $5 \alpha$-Pro [4 (2\%) IgG, $1(0.5 \%)$ IgM], 3 anti- $\beta_{2}$ GPI [3 (1.5\%) IgG, no IgM] and 4 aCL [3 (1.5\%) IgG, $1(0.5 \%) \operatorname{IgM}]$. The most frequent APA was $\alpha$-Pro $\operatorname{IgG}$ and the least frequent was anti$\beta_{2}$ GPI IgM as no controls had significant levels of this antibody.

\section{ET Patients}

Twenty of the 68 (29\%) ET patients studied had significant levels of at least one of the APA, these results are shown in Table 1. Most of these antibodies were of the IgM subclass 21/27 (78\%). Overall 2/68

Table 1 Summary of APA positive ET patients

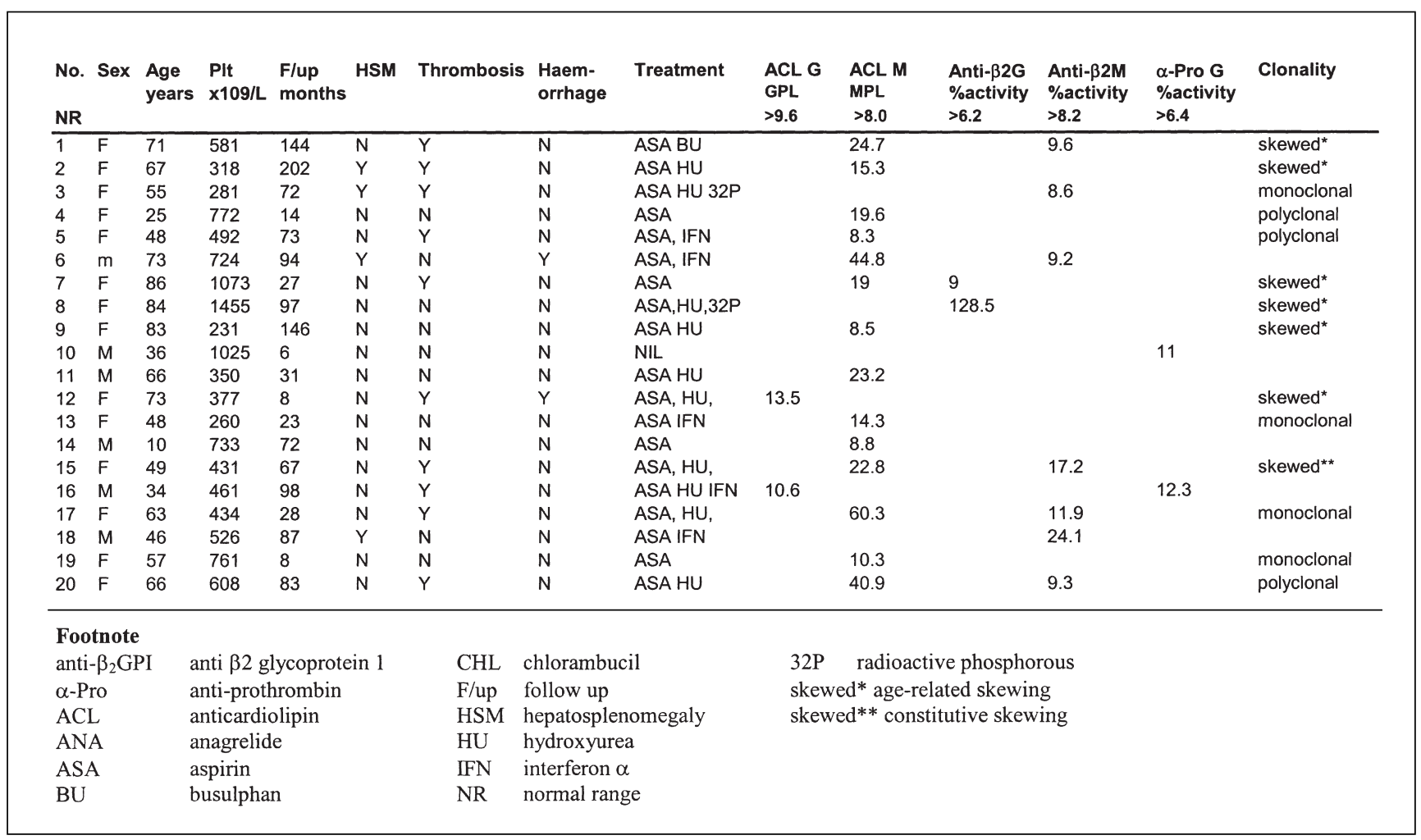


$\alpha$-Pro (all IgG), 9/68 anti- $\beta_{2}$ GPI (2/68 IgG, 7/68 IgM) and 16/68 aCL $(2 / 68 \mathrm{IgG}, 14 / 68 \mathrm{IgM})$. Of the aCL IgM antibodies three were of low titre (<10 MPL), five antibodies were 10-20 MPL, three 20-40 MPL and three greater than $40 \mathrm{MPL}$. Seven of the ET patients had a combination of two positive APA results and 13 had a single class of APA detected. A summary of the thrombotic events in APA positive and negative ET patients is given in Table 2. As the samples were tested retrospectively the results are from a single time point only and the majority of patients were not retested. Repeat assays were however performed upon seven patients and remained positive at similar titre in six, the patient (number 5, Table 1) whose APA did not demonstrate persistence had low titre aCL IgM.

\section{Comparison of the Prevalence of APA in Normal Controls and ET Patients}

Previous studies have suggested that there might be an increased incidence of APA with age (10). Therefore a control group for this study was specifically selected that consisted of healthy older subjects. There was a difference between the age of the ET patients and "healthy elderly" controls who were significantly older $(p=0.01$, Mann-UWhitney test). Despite the older age of the control population the prevalence of APA in patients with ET appeared to be greater than in normal controls with 27 positive screens compared to 12 in controls ( $p<0.0001)$. When the different subclasses of APA were individually analysed this difference was significant for aCL IgM and anti- $\beta_{2}$ GPI $\operatorname{IgM}(\mathrm{p}<0.0001$ for both).

\section{Comparison of Clinical Details of ET Patients with and without Detectable APA}

The clinical records of all patients were examined to determine whether there were any differences between those patients with and without detectable APA (Table 3) The only parameter which was significant between the two groups of patients was the occurrence of thrombotic events. Overall 10/20 (50\%) of those ET patients with detectable APA had thrombotic events, in comparison 12/48 (25\%) of patients with no detectable APA had thrombotic events. This difference in the prevalence of thrombotic events reaches statistical significance $(\mathrm{p}=0.04)$. The relative risk of thrombosis in the APA positive patients compared to the APA negative patients was $2.0,95 \%$ confidence intervals 1.03-3.86. The confidence intervals are wide reflecting the small number of patients. The incidence of thrombosis in the APA negative group was 5.3/100 patient-years and 8.70/100 patient-years in the APA positive group; most of the thrombotic events however had occurred at or after the time of diagnosis so this analysis may not reflect the true increase in risk.

The thrombotic events in the APA positive and negative patients are shown in Table 2. The distribution of the site of thrombosis, arterial or venous, was similar between APA positive and negative patients. Although there appears to be a greater prevalence of transient ischaemic attack in the APA negative group, and of recurrent miscarriage and stroke in the APA positive group, these differences are not significant. Detailed analysis of these thrombotic events revealed that in 8/20 APA positive and 9/48 APA negative ET patients thrombosis had already occurred at the time of diagnosis. Overall 2/20 ET patients with detectable APA had multiple thromboses in comparison with 2/48 APA negative patients, which was not statistically significant although the number of patients and events is probably too small to demonstrate a difference. Furthermore, multiple antibodies were associated with greater risk of a thrombotic event $4 / 13$ with single subclass had thrombosis, not a significant association, whereas $6 / 7$ with two subclasses of APA had thrombotic events. The prevalence of thrombosis in patients with two APA was statistically significant when compared to those patients with either a single APA or no detectable APA ( $p=0.02$, and $\mathrm{p}<0.0002$ respectively). Although the number of patients is relatively small there was still a suggestion that anti- $\beta_{2}$ GPI IgM alone or in combination was a greater risk for thrombosis. Overall 5/7 patients who had detectable anti- $\beta_{2}$ GPI IgM had thrombosis, compared to $5 / 13$ with other APA, or $17 / 61$ of the ET patients with no anti- $\beta_{2}$ GPI IgM detected $(p=N S$ and $p=$ 0.02 respectively).

\begin{tabular}{|c|c|c|}
\hline \multicolumn{2}{|c|}{$\begin{array}{r}\text { APA positive* } \\
\text { no (\%) }\end{array}$} & \multirow[t]{2}{*}{$\begin{array}{r}\text { APA negative * } \\
\text { no }(\%)\end{array}$} \\
\hline Major thrombotic events & & \\
\hline DVT/PE & $1(5)$ & $1(2)$ \\
\hline Multiple DVT & $1(5)$ & $2(4)$ \\
\hline \multicolumn{3}{|l|}{ Portal vein thrombosis } \\
\hline CVA & $2(10)$ & $1(2)$ \\
\hline Myocardial infarct & $1(5)$ & \\
\hline Retinal vein thrombosis & $1(5)$ & $1(2)$ \\
\hline Digital thrombosis & $1(5)$ & \\
\hline \multicolumn{3}{|l|}{ Minor thrombotic events } \\
\hline Erythromelalgia & $1(5)$ & $3(6)$ \\
\hline Transient ischaemic attack & $2(10)$ & $4(8)$ \\
\hline Recurrent miscarriage & $1(5)$ & \\
\hline Thrombosis at/or before diagnosis & $8(40)$ & $9(18)$ \\
\hline Multiple thrombotic events & $2(10)$ & $2(4)$ \\
\hline * Some patients had more than one $\mathrm{e}$ & & \\
\hline
\end{tabular}

Table 2 Summary of thrombotic events in the ET patients 
Table 3 Comparison of the clinical features in APA positive and negative ET patients

\begin{tabular}{|c|c|c|c|}
\hline & $\begin{array}{l}\text { Positive antiphospholipid } \\
\text { antibody screen } \\
(\mathrm{n}=20)\end{array}$ & $\begin{array}{c}\text { Negative antiphospholipid } \\
\text { antibody screen } \\
(\mathrm{n}=48)\end{array}$ & Difference* \\
\hline $\begin{array}{c}\text { Median age at time } \\
\text { of test }\end{array}$ & 63 years & 52 years & NS \\
\hline $\begin{array}{l}\text { Median plt count at } \\
\text { test }(\mathrm{x} 109 / \mathrm{L})\end{array}$ & $526 \times 10^{9} / \mathrm{L}$ & $488 \times 10^{9} / \mathrm{L}$ & NS \\
\hline Newly diagnosed & 3 & 7 & NS \\
\hline $\begin{array}{l}\text { Median follow up } \\
\text { (months) }\end{array}$ & 72 & 34 & NS \\
\hline $\begin{array}{c}\begin{array}{c}\text { Mean follow up } \\
\text { (months) }\end{array} \\
\end{array}$ & 69.1 & 56.6 & NS \\
\hline $\begin{array}{c}\text { Hepato- } \\
\text { splenomegaly }\end{array}$ & 4 & 8 & NS \\
\hline Thrombosis & 10 & 12 & $\mathrm{P}=0.04$ \\
\hline Haemorrhage & 2 & 4 & NS \\
\hline Aspirin alone & $4 * *$ & 17 & NS \\
\hline $\begin{array}{c}\text { Cytoreductive } \\
\text { treatment }+ \text { aspirin }\end{array}$ & 15 & 31 & NS \\
\hline $\begin{array}{c}\text { Incidence of } \\
\text { thrombosis } / 100 \\
\text { patient-years }\end{array}$ & 8.7 & 5.3 & NS \\
\hline \multicolumn{4}{|c|}{$\begin{array}{l}\text { * Chi square test applied to all except Mann-U-Whitey was used to compare platelet } \\
\text { count and follow up. } \\
\text { ** One patient was not given cytoreductive treatment or aspirin }\end{array}$} \\
\hline
\end{tabular}

\section{Correlation between APA Assays with Results of Clonal Analysis} in ET Patients

Altogether 44 females were evaluable for clonality assays; results were unassessable for 19 patients; due to possible age-related skewing in 13, or constitutive skewing in six. Monoclonal or polyclonal haemopoiesis did not appear to correlate with APA. Ten females had monoclonal haemopoiesis, 4/10 had APA of these two had thrombosis. Twelve were polyclonal, $3 / 12$ had detectable APA and of these patients 2/3 had thrombotic episodes.

\section{Discussion}

The aetiology of both arterial and venous thrombosis and the pathogenic role of many prothrombotic conditions are complex and not well understood even in patients who are haematologically normal. Here we investigated the prevalence of APA in ET and their association with the thrombotic complications of this disease. We have been able to extend and confirm our original description of the apparent association between immunologically detectable APA and ET (8). Testing for the lupus anticoagulant was not performed in all patients as they were screened retrospectively, and some had only been reviewed in our centre on a single occasion with clinical follow up occurring locally. There was a very significant increase in the prevalence of aCL IgM and anti- $\beta_{2}$ GPI IgM when compared to that for 200 "elderly" normal controls ( $p<0.0001$ for each) thus strengthening our original suggestion of an association between APA and ET.

The preponderence of IgM class APA identified in our ET patients is striking. There is considerable ongoing debate as to which type of APA are the best markers of the antiphospholipid syndrome and hence of pathological significance $(10,16)$. Traditionally IgM antibodies may have been regarded as of lesser significance than those of IgG subclass.
However in a retrospective study of patients with systemic lupus erythematosis (SLE) mutlivariate analysis demonstrated that IgM ACL did correlate with venous but not arterial thrombosis (17). Furthermore we recently published data suggesting that aCL IgM and anti- $\beta_{2} \mathrm{GPI}$ $\operatorname{IgM}$ in patients with primary antiphospholipid syndrome and SLE are significantly associated with thrombosis $(\mathrm{p}<0.0001$ and $\mathrm{p}=0.011$ respectively) (15).

The pattern of detectable APA was not the same for each of the patients with ET. The majority of patients had aCL IgM (16/20) and most $(7 / 20)$ of those who had dual positive APA had both aCL and anti- $\beta_{2}$ GPI. It is interesting to note that two patients (nos. 8 and 18) had anti- $\beta_{2}$ GPI in the absence of aCL, this may relate to species specificity since bovine $\beta_{2}$ GPI is used in the aCL assays. Only one patient (no. 10) had $\alpha$-Pro alone interestingly this patient suffered both a myocardial infarction and a cerebrovascular accident. In a previous study of middle-aged men with myocardial infarction a high prevalence of $\alpha$-Pro was reported (18).

The association between APA and haematological malignancies has already been documented. Finazzi and colleagues (19) reported the natural history of 360 patients with APA documenting an increased estimated incidence of non-Hodgkin's lymphoma ( $0.28 \%$ per patient year). There are also reports of APA in patients with newly diagnosed non-Hodgkin's lymphoma and acute myeloid leukaemia $(20,21)$. It could be hypothesised that patients who form APA have a disordered immune surveillance system, perhaps permitting the emergence of a malignant disease like ET, or non-Hodgkin's lymphoma. This would seem unlikely in ET as these patients have not hitherto displayed any features suggestive of impaired immune surveillance. Furthermore only 1 of the 68 ET patients reported here fulfilled diagnostic criteria for autoimmune disease (rheumatoid arthritis) and this patient did not have detectable APA. 
Patients with ET might be more likely to form APA as a secondary phenomenon for a number of reasons including that their platelet membranes have been shown to have a number of abnormalities for example, an increased negative charge (22). This is potentially relevant as $\beta_{2}$ GPI binds to anionic phospholipids subsequently undergoing a conformational change which exposes epitopes for certain APA subtypes. Prolonged or abnormal platelet activation or formation of platelet-leucocyte complexes, might trigger APA formation due to the chronic exposure of phospholipid binding proteins like $\beta_{2}$ GPI and prothrombin. Both enhanced platelet activation and platelet-leucocyte complexes have been demonstrated in some patients with APS (23) and ET (24-26). Polymorphonuclear leucocyte activation has also been demonstrated in ET in association with activation of the endothelium and coagulation system (27). APA, especially anti- $\beta_{2}$ GPI, are known to bind to endothelial cells (28). The presence of endothelial damage, due to leucocyte activation, or within the bone marrow sinusoids themselves due to the increased number of megakaryocytes, might trigger anti- $\beta_{2}$ GPI formation. Clonal analysis suggests that ET is heterogeneous with a significant proportion of polyclonal patients $(5,6)$. The relationship between results of clonality assays and APA prevalence was examined here and there appeared to be no clear correlation. Nevertheless only a limited number of informative females were analysed and the possible relationship between clonal status and APA merit future study.

Antiphospholipid antibodies are known to arise as a secondary phenomenon following exposure to certain drugs, might hydroxyurea be capable of triggering the same response? Detailed analysis of this data would suggest that this is probably not the case as ten of the patients with significant APA had not been treated with hydroxyurea at any time. Furthermore four of these ET patients were found to have detectable APA whilst being treated with aspirin alone (3) or nothing (1). Finally there is a well-established relationship between both recent thrombosis or infection and transient positive APA [reviewed in (29)]. Patients with ET are however not especially susceptible to infections and all our patients were well and thrombosis free for at least three months at the time the assays were performed.

From the data presented here it would appear to be unlikely that APA are merely an innocent epiphenomenon in ET. The significance of the data is however limited by the single point prevalence design of the study, and lack of measurement of lupus anticoagulant activity. There was a significant association between APA and occurrence of thrombosis in ET ( $p=0.04$ overall and $p<0.0002$ for those with two detectable APAs). The relative risk of thrombosis associated with APA positive results was 2.0 (95\% confidence interval 1.03-3.86), the confidence intervals reflecting the limited patient numbers in this study. There was also a suggestion of a greater influence of anti- $\beta_{2}$ PI upon thrombotic risk $(p=0.02)$. An apparent increase in thrombotic events could be due in part to a different time at risk (follow up). Therefore it is important that although the median follow up for the two groups of patients was different (72 vs. 34 months), mean follow up for the two groups was similar (69.1 vs. 56.6 months) and there was no statistical difference in length of follow up. The incidence of thrombosis was 8.70/100 patientyears and 5.3/100-patient-years in the APA positive and negative groups respectively. However as a large proportion of the thrombotic events occurred at or before diagnosis (Table 2) this means of expressing thrombotic risk with detectable APA may be less appropriate. Unfortunately there is insufficient evidence to suggest any correlation between antibody titre and thrombosis, nor between APA in ET and either timing, site or number of thrombotic events. Proposed mechanisms for the prothrombotic state in antiphospholipid syndrome include persistent activation of the coagulation cascade, inhibition of the anticoagulant actions of protein $\mathrm{C}$ activation and its inhibition of fibrinolysis and direct platelet activation (30). Further evaluation of these and the other potential mechanisms for APA promoting thrombosis in ET are warranted.

This report should initiate further investigation into the relationship between APA and ET and other MPDs as well as providing potential insight into the mechanism of thrombosis in both ET and antiphospholipid antibody syndrome. The observation of an increased prevalence of thrombotic complications in ET patients, associated in particular with anti- $\beta_{2}$ GP1 or multiple APA, is important and if confirmed in larger studies might influence patient management.

\section{References}

1. Cortelazzo S, Finazzi G, Ruggeri M, Vestri O, Galli M, Rodeghiero F, Barbui T. Hydroxyurea for patients with essential thrombocythemia and a high risk of thrombosis. N Engl J Med 1995; 332: 1132-6.

2. Lengfelder E, Hochhaus A, Kronawitter U, Hoche D, Queisser W, JahnEder M, Burkhardt R, Reiter A, Ansari H, Hehlmann R. Should a platelet limit of $600 \times 10(9) / 1$ be used as a diagnostic criterion in essential thrombocythaemia? An analysis of the natural course including early stages. Br J Haematol 1998; 100: 15-23.

3. Sterkers Y, Preudhomme C, Lai JL, Demory JL, Caulier MT, Wattel E, Bordessoule D, Bauters F, Fenaux P. Acute myeloid leukemia and myelodysplastic syndromes following essential thrombocythemia treated with hydroxyurea: high proportion of cases with 17p deletion. Blood 1998; 91: 616-22.

4. Cortelazzo S, Viero P, Finazzi G, D’Emilio A, Rodeghiero F, Barbui T. Incidence and risk factors for thrombotic complications in a historical cohort of 100 patients with essential thrombocythemia. J Clin Oncol 1990; 8: 556-62.

5. Harrison CN, Gale RE, Machin SJ, Linch DC. A large proportion of patients with a diagnosis of essential thrombocythaemia do not have a clonal disorder and may be at lower risk of thrombotic complications. Blood 1999; 93: 417-24.

6. Chiusolo P, La Barbera EO, Laurenti L, Piccirillo N, Sora F, Giordano G, Urbano R, Mazzucconi MG, De Stefano V, Leone G, Sica S. Clonal hemopoiesis and risk of thrombosis in young female patients with essential thrombocythemia. Exp Hematol 2001; 29: 670-6.

7. Juvonen E, Ikkala E, Oksanen K, Ruutu T. Megakaryocyte and erythroid colony formation in essential thrombocythaemia and reactive thrombocytosis: diagnostic value and correlation to complications. $\mathrm{Br} \mathrm{J}$ Haematol 1993; 83: 192-7.

8. Harrison CN, Machin SJ. Essential thrombocythaemia and antiphospholipid antibodies. Am J Med 1997; 102: 317-8.

9. Murphy S, Peterson P, Iland H, Laszlo J. Experience of the Polycythemia Vera Study Group with essential thrombocythemia: a final report on diagnostic criteria, survival, and leukemic transition by treatment. Semin Hematol 1997; 34: 29-39.

10. Petri M. Epidemiology of the antiphospholipid antibody. Journal of Autoimmunity 2000; 15: 145-51.

11. Scurr JH, Machin SJ, Bailey-Kig S, Mackie IJ, McDonald S, Smith PD. Frequency and prevention of symptomless deep-vein thrombosis in longhaul flights: a randomised trial. Lancet 2001; 357: 1485-9.

12. Loizou S, McCrea JD, Rudge AC, Reynolds R, Boyle CC, Harris EN. Measurement of anti-cardiolipin antibodies by an enzyme-linked immunosorbent assay (ELISA): standardization and quantitation of results. Clin Exp Immunol 1985; 62: 738-45.

13. Harris EN, Hughes GR. Standardising the anti-cardiolipin antibody test. Lancet 1987; 1: 77.

14. McNally T, Mackie IJ, Machin SJ, Isenberg DA. Increased levels of beta 2-glycoprotein-I antigen and beta 2-glycoprotein-I binding antibodies are 
associated with a history of thromboembolic complications in patients with SLE and primary antiphospholipid syndrome. Br J Rheumatol 1995; 34: 1031-6.

15. Donohoe S, Mackie IJ, Isenberg D, Machin SJ. Anti-prothrombin antibodies: assay conditions and clinical associations in the anti-phospholipid syndrome. Br J Haematol 2001; 113: 544-9.

16. Carreras LO, Forastiero RR, Martinuzzo ME. Which are the best biological markers of the antiphospholipid syndrome? Journal of Autoimmunity 2000; 15: 163-72.

17. Horbach DA, Oort EV, Donders RCJM, Derksen RHWM, deGroot PG, Lupus anticoagulant is the strongest risk factor for both venous and arterial thrombosis in patients with SLE. Thromb Haemost 1996; 76: 916-24.

18. Varaala O, Puurunen M, Manttari M, Manien V, Aho K, Palosuo T. Antibodies to prothrombin imply a risk of myocardial infarction in middle aged men. Thromb Haemost 1996; 75: 456-9.

19. Finazzi G, Brancaccio V, Moia M, Ciaverella N, Mazzucconi MG, Schinco PC, Ruggeri M, Pogliani EM, Gamba G, Rossi E, Baudo F, Manotti C, D'Angelo A, Palareti G, De Stefano V, Berrettini M, Barbui T. Natural history and risk factors for thrombosis in 360 patients with antiphospholipid antibodies: a four-year prospective study from the Italian Registry. Am J Med 1996; 100: 530-6.

20. Stasi R, Stipa E, Masi M, Oliva F, Sciarra A, Perrotti A, Zaccari G, Papa G. Antiphospholipid antibodies: prevalence, clinical significance and correlation to cytokine levels in acute myeloid leukemia and non-Hodgkin's lymphoma. Thromb Haemost 1993; 70: 568-72.

21. Stasi R, Stipa E, Masi M, Oliva F, Olivieri M, Perrotti A, Zaccari G, Amadori S, Papa G. Antiphospholipid antibodies: their prevalence, clinical significance and correlation with cytokine levels in acute myeloid leukemia and non-Hodgkin's lymphoma. Recenti Prog Med 1995; 86: 57-62.

22. Crook M, Machin S, Crawford N. Electrokinetic behaviour and surface sialic acid status of blood platelets in essential thrombocythaemia (ET). Eur J Haematol 1992; 49: 128-32.
23. Joseph JE, Harrison P, Mackie IJ, Isenberg DA, Machin SJ. Increased circulating platelet-leukocyte complexes and platelet activation in patients with antiphospholipid syndrome (APS), systemic lupus erythematosus (SLE) and rheumatoid arthritis (RA). Br J Haematol 2001; 115: 451-9.

24. Griesshammer M, Beneke H, Nussbaumer B, Grunewald M, Bangerter M, Bergmann L. Increased platelet surface expression of P-selectin and thrombospondin as markers of platelet activation in essential thrombocythaemia. Thromb Res 1999; 96: 191-6.

25. Jensen MK, de Nully Brown P, Lund BV, Nielsen OJ, Hasselbalch HC. Increased circulating platelet-leukocyte aggregates in myeloproliferative disorders is correlated to previous thrombosis, platelet activation and platelet count. Eur J Haematol 2001; 66: 143-51.

26. van Genderen PJ, Prins FJ, Michiels JJ, Schror K. Thromboxane-dependent platelet activation in vivo precedes arterial thrombosis in thrombocythaemia: a rationale for the use of low-dose aspirin as an antithrombotic agent. Br J Haematol 1999; 104: 438-41.

27. Falanga A, Marchetti M, Evangelista V, Vignoli A, Licini M, Balicco M, Manarini S, Finazzi G, Cerletti C, Barbui T. Polymorphonuclear leukocyte activation and hemostasis in patients with essential thrombocythaemia and polycythemia vera. Blood 2000; 96: 4261-6.

28. Hill MB, Phipps JL, Hughes P, Greaves M. Anti-endothelial cell antibodies in primary antiphospholipid syndrome and SLE: patterns of reactivity with membrane antigens on microvascular and umbilical venous cell membranes. Br J Haematol 1998; 103: 416-21.

29. Gharavi AE, Pierangeli SS. Infections and Antiphospholipid Antibodies. In: Khamashta M (ed) 2000. Hughes Syndrome Antiphospholipid Syndrome. London: Springer-Verlag Limited; pp 135-43.

30. Greaves M, Cohen H, MacHin SJ, Mackie I. Guidelines on the investigation and management of the antiphospholipid syndrome. Br J Haematol 2000; 109: 704-15.

Received July 9, 2001 Accepted after resubmission February 4, 2002 